\title{
Recent advances of glucocorticoids in the treatment of Duchenne muscular dystrophy (Review)
}

\author{
TIANYUAN ZHANG and XIANGDONG KONG
}

Center of Prenatal Diagnosis, The First Affiliated Hospital of Zhengzhou University, Zhengzhou, Henan 450052, P.R. China

Received July 17, 2020; Accepted December 18, 2020

DOI: $10.3892 /$ etm.2021.9875

\begin{abstract}
Duchenne muscular dystrophy (DMD) is the most common degenerative neuromuscular disease. The incidence of DMD in live births is $1 / 3,600-1 / 6,000$. Although glucocorticoid-dependent medication is the mainstay treatment option for DMD, a standard treatment regimen has yet to be determined. The present review discusses the literature on the timing, methods and courses of glucocorticoid treatment for DMD. The review highlights the importance of the immediate commencement of glucocorticoid treatment following the diagnosis of DMD, with weekend-only administration being advantageous. Adherence to long-term single-glucocorticoid therapy can delay the loss of ambulation ability, and the side effects of the treatment are controllable. However, the standard medication for patients of different ages and stages of disease development, and the use of combination therapy require further investigation.
\end{abstract}

\section{Contents}

1. Introduction

2. Mechanism of action of glucocorticoids in the treatment of DMD

3. Clinical application of glucocorticoids in the treatment of DMD

4. Adverse reactions of glucocorticoids in the treatment of DMD

5. Other drug treatments for DMD

6. Conclusions

Correspondence to: Professor Xiangdong Kong, Center of Prenatal Diagnosis, The First Affiliated Hospital of Zhengzhou University, 1 Jianshe East Road, Erqi, Zhengzhou, Henan 450052, P.R. China E-mail: kongxd@263.net

Key words: Duchenne muscular dystrophy, glucocorticoid, medical treatment

\section{Introduction}

Duchenne muscular dystrophy (DMD) is the most common degenerative neuromuscular disease and the most prevalent $\mathrm{x}$-linked recessive genetic disease, with an incidence of 1/3,600-1/6,000 in live births (1). DMD is caused by the deficiency of dystrophin, an anti-dystrophy protein encoded by the DMD gene, which leads to the progressive degeneration of muscle fiber and muscle necrosis (2). DMD usually occurs in childhood and has no effective cure. Patients with DMD generally lose their ability to walk when $\sim 12$ years old, and often require the use of a wheelchair. To date, numerous studies have demonstrated the efficacy of glucocorticoids in the treatment of DMD. Glucocorticoids have been shown to prolong autonomous walking, reduce scoliosis, and improve cardiopulmonary function, quality of life and the survival rate of patients (3). Despite their efficacy, the clinical use of glucocorticoids for DMD is known to be associated with adverse reactions, including osteoporosis, obesity, short stature, delayed puberty and adrenal insufficiency. The present review discusses the mechanisms of action of glucocorticoids in the treatment of DMD, and highlights advances in the management of DMD.

\section{Mechanism of action of glucocorticoids in the treatment of DMD}

Muscle biopsies of patients with DMD exhibit severe muscle fiber degeneration, necrosis and regeneration, as well as chronic inflammatory reactions, which can be antagonized by the anti-inflammatory effect of glucocorticoids (4). In addition, endothelial dysfunction can affect the perfusion of contracting muscles, resulting in progressive intramuscular ischemia and exacerbated endothelial dysfunction. Hörster et al demonstrated that glucocorticoids regulate the L-arginine/nitric oxide pathway in DMD and increase nitric oxide synthesis, thereby improving endothelial function and alleviating muscle ischemia or hypoxia (5).

Osteopontin (OPN), encoded by the secreted phosphoprotein 1 (SPP1) gene, plays a major role in the pathology of DMD by modulating muscle inflammation and regeneration. A polymorphism in the SPP1 promoter (rs28357094) has been shown to modify the response of DMD to glucocorticoid treatment. Vianello et al (6) reported that the rs $28357094 \mathrm{G}$ allele of the SPP1 promoter induces the expression of OPN in the presence 
of deflazacort, and regulates the inflammation and regeneration of muscle tissues. In addition, McRae et al (7) identified a new gene target, versican, that is associated with muscle regeneration in DMD. They investigated the expression and processing of versican in the hindlimb and diaphragm muscles of dystrophin-deficient ( $m d x$ ) and C57BL/10 wild-type (WT) mice. The mRNA and protein levels of the V0 and V1 splice variants of versican (Vcan) were found to be upregulated in the muscles of the $m d x$ mice compared with the WT mice, particularly in the diaphragm. However, following glucocorticoid treatment, a $50 \%$ downregulation of Vcan was observed. In addition, the glucocorticoid dexamethasone was demonstrated to promote myoblast fusion, which plays a key role in muscle regeneration, by inhibiting the expression of the Vcan gene.

The use of glucocorticoids to treat DMD induces some adverse hormonal reactions, which are associated with glucocorticoid receptor-mediated transactivation (8). In addition, the ergogenic effects of glucocorticoids are mediated by the direct induction of the metabolic transcription factor Krüppel-like factor 15 (KLF15). This proergogenic metabolic effect underlines the therapeutic role of glucocorticoids in DMD, as muscle glucocorticoid-KLF15 signaling has been demonstrated to improve muscle performance in patients with DMD (8).

Dystrophin and its homolog utrophin are important cytoskeletal proteins, which prevent the induction of damage by abnormal muscle contraction and ameliorate the clinical manifestations of DMD (9). Prednisone has been shown to increase the expression of dystrophin and utrophin proteins in healthy individuals and patients with DMD (10).

In a study involving 9 boys with DMD, aged $6 \pm 2$ years, the mRNA transcripts associated with satellite cell activation, myogenesis, regeneration, adipogenesis, muscle growth and tissue inflammation in muscle biopsies, serum creatine kinase $(\mathrm{CK})$ levels and muscle protein expression levels were compared before and after deflazacort treatment. The data showed that although the glucocorticoid treatment had no significant effect on the expression of muscle protein, the levels of inflammatory factors in the muscle tissues and the serum level of CK decreased. These results indicate that the effective therapeutic effect of glucocorticoids is mediated via numerous regulatory pathways (11).

\section{Clinical application of glucocorticoids in the treatment of DMD}

Timing of medication. Previous studies have demonstrated that it is beneficial to start using glucocorticoids in the early stages of DMD development, even before clinical symptoms become evident $(12,13)$. In a review of a series of 465 cardiac MRI studies of patients with DMD, the left ventricular ejection fraction (LVEF) and presence of late gadolinium enhancement (LGE), a marker for myocardial fibrosis, were examined. An age-based analysis revealed that LVEF decreased by $0.58 \pm 0.10 \%$ per year, with a greater reduction of $2.2 \pm 0.31 \%$ per year observed in the patients with LGE (14). Sawnani et al (15) reviewed the lung functions of 220 patients with DMD over a period of 6 years, and found nonlinear rates of decline, which accelerated with age. However, data from a national study on the molecular and clinical characteristics of children with DMD in Norway highlighted significant differences in disease progression, with diagnostic delays occurring in those presenting with late motor development (16). Therefore, although there is no definitive time point for the commencement of treatment in children with DMD, it is recommended that glucocorticoid administration begins immediately after diagnosis.

Medication scheme. Although the use of glucocorticoids is of benefit to patients with DMD, there is no standardized corticosteroid treatment dosage for DMD. However, a number of studies have evaluated different glucocorticoid therapy regimens for DMD, as documented in Table I.

Qi et al (17) retrospectively evaluated the diagnostic data of 155 children with DMD. Firstly, dexamethasone was intermittently administered by intravenous infusion at a dosage of 5-10 mg/day for 10-15 days according to body weight, age and the serum level of CK. Then, oral prednisone maintenance therapy at a dosage of $0.50-0.75 \mathrm{mg} / \mathrm{kg} /$ day was administered following 1 month of the pulse therapy. The data revealed that the serum level of CK after the low-dose hormone maintenance therapy was slightly higher than that after the pulse therapy, but remained lower than that prior to treatment. Notably, the follow-up data of $>9$-year-old children (22 cases in total) who chose to receive hormone therapy showed that 13 cases retained the ability to walk.

In a preliminary study of children with DMD in southwest China, it was found that $0.75 \mathrm{mg} / \mathrm{kg} /$ day prednisone slowed disease progression, improved quantitative muscle ultrasound results and the quality of life of the patients. In addition, the treatment was well tolerated with only moderate side effects (18). In another study, Wong et al (19) followed 97 adolescent patients with DMD who took daily glucocorticoids, predominantly deflazacort $(89 \%)$, with an mean treatment duration of 8.5 years. The study demonstrated that the patients' ability to exercise and cardiopulmonary functions were well preserved, and the side effects of the glucocorticoids were tolerable.

The effects of daily prednisolone use in patients with DMD have been reported in two Japanese studies. Sato et al (20) evaluated the effects of $0.75 \mathrm{mg} / \mathrm{kg}$ prednisolone on the intellectual ability and motor functions of 20 patients over a 2-year period. In the prednisolone-treated patients, an improvement in the intelligence quotient score was observed compared with that the control group, and motor functions were preserved. By contrast, Ishigaki et al (21) compared longitudinal clinical data between 7 patients treated with $0.5 \mathrm{mg} / \mathrm{kg}$ prednisolone every other day and 10 untreated patients, aged $>15$ years. They found that the age at which the loss of ambulation and initiation of noninvasive ventilation occurred did not differ between the treated and untreated patients. However, further assessment of the efficacy and safety of daily and every-other-day regimens is necessary, as the evidence on this is limited.

In a study by Connolly et al (22), 25 infants and young boys with DMD, aged 0.4-2.4 years, were treated with $5 \mathrm{mg} / \mathrm{kg}$ prednisolone twice a week for 1 year, and the changes in Bayley-III scales of infant development (Bayley-III) were compared with those of untreated boys at the 1-year follow-up. The data showed that twice weekly prednisolone increased the Bayley-III gross motor scale score of the children with 


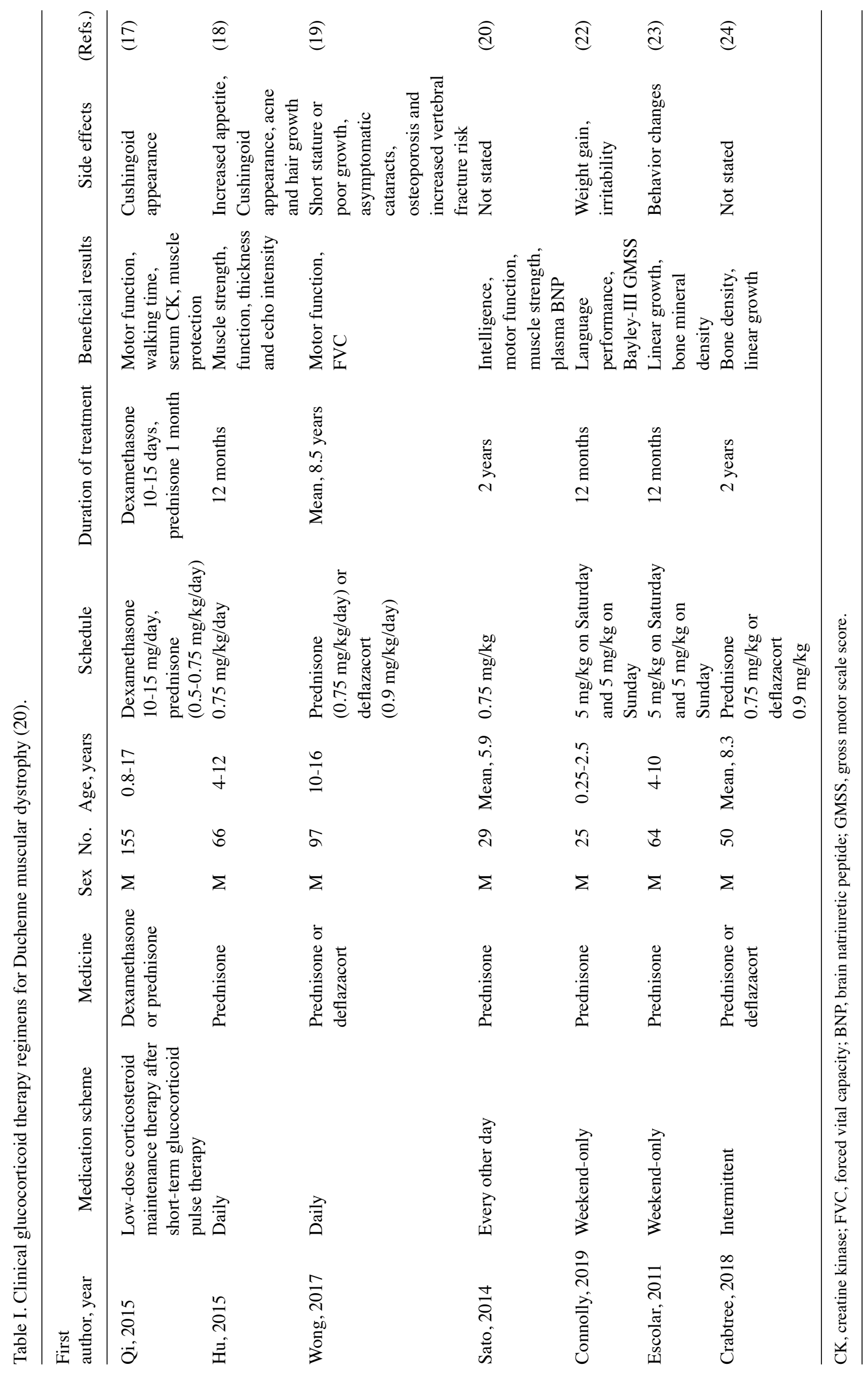


DMD. All the boys maintained linear growth and none of them developed Cushing syndrome. However, 13 of the 23 boys (56\%) who completed the study were overweight. A double-blind randomized controlled trial compared the effect of daily prednisone $(0.75 \mathrm{mg} / \mathrm{kg} /$ day $)$ with weekend-only prednisone $(5 \mathrm{mg} / \mathrm{kg} /$ weekend day) in the treatment of DMD. A total of 64 boys with DMD, aged between 4 and 10 years, were randomized at 12 centers of the Cooperative International Neuromuscular Research Group. The study data demonstrated that the weekend-only use of prednisone was as effective as daily prednisone for the 12-month evaluation period. No significant difference in muscle strength or function was detected between the two modes of administration. However, the children who adopted the weekend-only use of prednisone exhibited a greater linear increase in height (23).

In another study, Crabtree et al (24) evaluated the effect of a daily or intermittent glucocorticoid regimen, with 10 days on and 10 days off, in 50 boys with DMD from two centers. The data showed that while the boys who received the daily regimen were more ambulant, they had a higher incidence of vertebral fracture, obesity and short stature after 2 years of treatment. By contrast, the boys who took glucocorticoids intermittently had fewer vertebral fractures, but were less likely to walk independently. This study demonstrates the effectiveness of the intermittent regimen and its reduced side effects. An intermittent regimen is also considered to be the last treatment option prior to complete withdrawal.

Abutaleb et al (25) reported the case of a patient with DMD who suddenly stopped using deflazacort and switched to the same dose of prednisone, which resulted in increased myocardial inflammation, edema and fibrosis. However, after restarting the deflazacort regimen, an improvement in symptoms was observed. Therefore, the sudden withdrawal of a corticosteroid or change in the type of corticosteroid may lead to adverse reactions in patients with DMD treated with single or combined drugs, and the continuous use of a specific corticosteroid is particularly important.

Although daily glucocorticoid use has been shown to prolong the walking function of patients with DMD, the marked side effects have led to the exploration of different treatment regimens. These may reduce the toxicity and side effects of glucocorticoids and, in the case of the weekend-only regimen, exhibit an improved performance. In addition, during the course of treatment, it is also recommended to use glucocorticoids at different levels according to the disease progression and to not change the types of glucorticosteroids that are used.

Course of treatment. A recent study observed that after 3 months of treatment with deflazacort, the levels of inflammatory factors in muscle tissues and of CK in the blood of children with DMD declined (11). A phase III, double-blind, randomized, placebo-controlled multicenter study assessed the muscle strength of 196 boys, aged 5-15 years, with DMD. The muscle strength in the $0.9 \mathrm{mg} / \mathrm{kg} /$ day deflazacort, $1.2 \mathrm{mg} / \mathrm{kg} / \mathrm{day}$ deflazacort and $0.75 \mathrm{mg} / \mathrm{kg} /$ day prednisone treatment groups at 12 weeks was significantly higher than that of the placebo group (26). In addition, a previous study showed that the treatment of DMD with corticosteroids improved muscle strength and function for 12 months, and increased muscle strength for up to 2 years. Furthermore, patients continued to benefit for as long as 66 months, with common but controllable adverse reactions (27).

A multicenter prospective cohort study followed up pulmonary function indicators for 10 years in 322 patients with DMD who were treated with glucocorticoids for $>1$ year, and compared them with 53 patients who had received $<1$ month of treatment with glucocorticoids. The data showed that the long-term use of glucocorticoids slowed down the progression of lung disease in DMD throughout the lifespan of the patients (28). Another multicenter study that followed up patients with DMD for 10 years showed that receiving glucocorticoid treatment for $\geq 1$ year increased the median age of loss of mobility milestones from 2.1 to 4.4 years and that of upper limb milestones from 2.8 to 8.0 years. The risk of death was decreased significantly compared with that of patients who had either not received any glucocorticoid treatment or had a cumulative treatment duration of $<1$ month (3). Furthermore, Pane et al (29) found that the continuous use of glucocorticoids after the loss of walking ability in adolescents and adults was beneficial to upper limb function.

The present review indicates that patients with DMD may gain clinical benefits after receiving glucocorticoid treatment for 3 months, with 10-year follow-ups confirming that the long-term use of glucocorticoids improves cardiopulmonary function, preserves ambulation and prolongs the survival of patients. However, since great differences exist in the disease progression of DMD among patients, the specific regimen should vary from person to person. In addition, it may be necessary to apply glucocorticoid therapy for DMD as a long-term treatment.

\section{Adverse reactions of glucocorticoids in the treatment of DMD}

The present review identified that growth disorders, delayed puberty, obesity, osteoporosis or fracture, cataract and adrenal insufficiency are some of the side effects associated with the long-term use of glucocorticoids in the treatment of DMD. The prevention of obesity is important for the long-term management of patients with DMD, and is the main reason for drug withdrawal (30). Most of the adverse reactions of glucocorticoids in the treatment of DMD occur after 6 months of treatment, but can be closely monitored and prevented (31).

The main manifestations and management (31): Obesity may be controlled by emphasizing the importance of weight control and providing a healthy diet plan prior to medication, while the acne and skin rashes that occur frequently in puberty may be treated with topical drugs, without adjustment of the glucocorticoid treatment. Height should be measured regularly, preferably at least once every 6 months, and the level of glucocorticoid evaluated if growth stagnation is observed. Behavioral abnormalities mostly appear in the first 6 weeks of glucocorticoid use but are alleviated over time and, like increased hair growth, do not require any adjustment of medication. The presence of myoglobinuria, indicated by abnormally colored urine after exercise, may be managed by avoiding excessive muscle stretching and high resistance movements. With regard to serious infection 
in immunosuppressed patients, all appropriate vaccinations should be administered before starting treatment, with adjustment of the glucocorticoid dose during stress, such as fever or surgery. To avoid abrupt drug withdrawal, monitoring changes in blood pressure, administering a low-salt diet to lower blood pressure, and angiotensin-converting enzyme inhibitors or $\beta$-receptor blockers is recommended. In addition, abnormal glucose metabolism and digestive tract symptoms such as gastritis, abdominal pain and hematochezia should be monitored so as to avoid the use of non-steroidal anti-inflammatory drugs. Annual bone mineral density tests, blood 25-hydroxyvitamin D level monitoring, weight-bearing exercise and concurrent calcium and vitamin D supplements are recommended. Annual ophthalmology examinations and the timely detection of cataracts affecting vision are also encouraged.

Indicators that the glucocorticoid dosage should be reduced include a $>10 \%$ increase in body mass index for $>3$ months, fasting blood glucose levels $>100 \mathrm{mg} / \mathrm{dl}$ after diet control, increased diastolic blood pressure that exceeds the upper limit of the normal age group by $10 \mathrm{mmHg}$, increased systolic blood pressure $>15 \mathrm{mmHg}$ after 1 month on a low-salt diet, and other untreatable adverse drug reactions (32).

A number of studies have evaluated alternative evaluation indicators in order to better assess the benefits of administering glucocorticoids to patients with DMD and identify adverse drug reactions in a timely manner. For example, Al-Zougbi et al (33) suggested the use of bone age to evaluate the bone mineral density of patients with DMD, and lateral $\mathrm{X}$-ray films of the spine have been used as part of routine bone health monitoring in patients with DMD following the initiation of a glucocorticoid regimen, (34). In addition, Tandon et al (14) suggested the use of myocardial fibrosis load to predict LVEF and thereby evaluate cardiac function in patients with DMD. Furthermore, Sawnani et al (15) recommended the use of forced vital capacity (FVC) deciles to test the lung functions of patients with DMD.

\section{Other drug treatments for DMD}

Considering the adverse reactions caused by the long-term use of common corticosteroids, including dexamethasone, prednisone, deflazacort and hydrocortisone, research has focused on the development of new drugs, repurposing of known drugs and evaluation of the exact effects of the drugs commonly used in DMD therapy to combat adverse reactions.

In 2016, the US Food and Drug Administration granted accelerated approval for eteplirsen (Exondys 51), a drug that promotes dystrophin production by restoring the translational reading frame of DMD through exon 51 skipping in defective gene variants. The approval was based on an unpublished, open-label study of 13 boys who were on a stable dose of corticosteroids for $\geq 6$ months and treated with eteplirsen (30 mg/kg) weekly for 48 weeks, after which 1 subject was unavailable for analysis. A median increase of $0.1 \%$ in dystrophin level was detected after treatment with eteplirsen for 48 weeks, resulting in a dystrophin level $0.22-0.32 \%$ of the normal value. However, this minimal increase in dystrophin, the surrogate endpoint of the study, does not assure a clinical benefit (35). Another drug, vamorolone, was evaluated in a 2-week open-label phase IIa trial with multiple ascending doses of $0.25,0.75,2.0$ and $6.0 \mathrm{mg} / \mathrm{kg} /$ day. The results indicated that vamorolone was safer than glucocorticoids. It was characterized by reduced insulin resistance and beneficial changes in bone turnover, with loss of increased bone resorption and decreased bone formation only at the highest dose, as well as reduced adrenal inhibition. As the first dissociative steroid to be developed, vamorolone has a preserved anti-inflammatory effect and reduced steroid-associated safety issues. The maximum tested dose of $6.0 \mathrm{mg} / \mathrm{kg} / \mathrm{day}$ is safe and well-tolerated (36).

Kumaki et al (37) reported the first known case of osteoporosis caused by glucocorticoid use in an adolescent with DMD that was successfully treated with denosumab. The report noted that lumbar bone mineral density and bone turnover were significantly improved following treatment with denosumab for 18 months, and no fractures or complications were recorded during the treatment. This suggests that denosumab merits further testing as an effective drug for the treatment of osteoporosis in patients with DMD. Tamixofen was evaluated for the treatment of DMD in a multicenter, randomized, placebo-controlled, double-blind phase 3 trial, which found that $20 \mathrm{mg}$ tamoxifen daily for 48 weeks slowed the progression of ambulant and non-ambulant DMD (38). In another phase 3 trial, the DMD Long-term Idebenone Study (DELOS) trial, idebenone was shown to reduce the loss of peak expiratory flow and FVC in patients with DMD in comparison with placebo (39). In addition, Victor et al (40) reported on a phase 3 clinical trial of 331 patients with DMD, aged 7-14 years, who were treated with glucocorticoid with or without tadalafil for 48 weeks. The results demonstrated that oral tadalafil once a day did not alleviate the decline in ambulatory ability in boys with DMD. However, further research is required to determine whether starting tadalafil treatment before the age of 7 years is able to slow the decline in ambulatory ability. The efficacy of most cross-disciplinary drugs is not clearly defined, and thus requires further study with long-term follow-ups.

Adolescents with DMD who are treated with long-term, high-dose glucocorticoids often experience severe puberty delays. Testosterone is the main circulating androgen in men, which promotes masculinity and growth (41). Wood et al (41) found that treatment with exogenous testosterone increased the height of adolescents with DMD by an average of $14.2 \mathrm{~cm}$ after 3.1 years. However, no standardized regimen was identified, and the effects of testosterone on muscle function and bone integrity in patients with DMD require further investigation. Perera et al (42) assessed the natural fracture history and vitamin D levels of patients with DMD, who are prone to osteoporosis and fractures. Despite long-term vitamin D supplementation, $38 \%$ of the patients were vitamin D deficient. However, Srinivasan et al (43) found that prophylactic oral bisphosphonate maintains bone mineral density, may reduce the incidence of fractures in patients with DMD who are treated with glucocorticoids, and is tolerated by the majority of patients.

\section{Conclusions}

The use of glucocorticoids can effectively delay the loss of ambulatory ability and prolong the life expectancy of patients 
with DMD. However, glucocorticoids have significant side effects, which has prompted the research and development of new drugs that may be used in combination therapy with glucocorticoids. At present, the clinical management of patients with DMD involves early detection and treatment to delay the progression of the disease, and the application of interventions based on the predicted side effects of glucocorticoids. Multidisciplinary comprehensive treatments and interdisciplinary nursing may be helpful to the prognosis of patients with DMD.

\section{Acknowledgements}

Not applicable.

\section{Funding}

National Key Research and Development Program of China (grant no. \#2018YFC1002203).

\section{Availability of data and materials}

Not applicable.

\section{Authors' contributions}

TZ wrote the manuscript and XK revised the main content of the manuscript. Both authors read and approved the final manuscript.

\section{Ethics approval and consent to participate}

Not applicable.

\section{Patient consent for publication}

Not applicable.

\section{Authors' information}

TZ is a postgraduate student at the Center of Prenatal Diagnosis, The First Affiliated Hospital of Zhengzhou University. XK is an employee of the Center of Prenatal Diagnosis, The First Affiliated Hospital of Zhengzhou University.

\section{Competing interests}

The authors declare that they have no competing interests.

\section{References}

1. Unguru Y: Ethical challenges in early-phase pediatric research for life-limiting illness. Semin Pediatr Neurol 22: 177-186, 2015.

2. Constantin B: Dystrophin complex functions as a scaffold for signalling proteins. Biochim Biophys Acta 1838: 635-642, 2014.

3. McDonald CM, Henricson EK, Abresch RT, Duong T, Joyce NC, Hu F, Clemens PR, Hoffman EP and Cnaan A: Long-term effects of glucocorticoids on function, quality of life, and survival in patients with Duchenne muscular dystrophy: A prospective cohort study. Lancet 391: 451-461, 2018.

4. Archer JD, Vargas CC and Anderson JE: Persistent and improved functional gain in mdx dystrophic mice after treatment with L-arginine and deflazacort. FASEB J 20: 738-740, 2006.
5. Hörster I, Weigt-Usinger K, Carmann C, Chobanyan-Jürgens K, Köhler C, Schara U, Kayacelebi AA, Beckmann B, Tsikas D and Lücke T: The L-arginine/NO pathway and homoarginine are altered in Duchenne muscular dystrophy and improved by glucocorticoids. Amino Acids 47: 1853-1863, 2015.

6. Vianello S, Pantic B, Fusto A, Bello L, Galletta E, Borgia D, Gavassini BF, Semplicini C, Sorarù G, Vitiello L and Pegoraro E: SPP1 genotype and glucocorticoid treatment modify osteopontin expression in Duchenne muscular dystrophy cells. Hum Mol Genet 26: 3342-3351, 2017.

7. McRae N, Forgan L, McNeill B, Addinsall A, McCulloch D, Van der Poel C and Stupka N: Glucocorticoids improve myogenic differentiation in vitro by suppressing the synthesis of versican, a transitional matrix protein overexpressed in dystrophic skeletal muscles. Int J Mol Sci 18: 2629, 2017.

8. Morrison-Nozik A, Anand P, Zhu H, Duan Q, Sabeh M, Prosdocimo DA, Lemieux ME, Nordsborg N, Russell AP, MacRae CA, et al: Glucocorticoids enhance muscle endurance and ameliorate Duchenne muscular dystrophy through a defined metabolic program. Proc Natl Acad Sci USA 112: E6780-E6789, 2015.

9. Manzur AY, Kuntzer T, Pike M and Swan A: Glucocorticoid corticosteroids for Duchenne muscular dystrophy. Cochrane Database Syst Rev: Jan 23, 2008. doi: org/10.1002/14651858. CD003725.pub3.

10. Baltgalvis KA, Call JA, Nikas JB and Lowe DA: Effects of prednisolone on skeletal muscle contractility in mdx mice. Muscle Nerve 40: 443-454, 2009.

11. Jensen L, Petersson SJ, Illum NO, Laugaard-Jacobsen HC, Thelle T, Jørgensen LH and Schrøder HD: Muscular response to the first three months of deflazacort treatment in boys with Duchenne muscular dystrophy. J Musculoskelet Neuronal Interact 17: 8-18, 2017.

12. Merlini L, Gennari M, Malaspina E, Cecconi I, Armaroli A, Gnudi S, Talim B, Ferlini A, Cicognani A and Franzoni E: Early corticosteroid treatment in 4 Duchenne muscular dystrophy patients: 14-year follow-up. Muscle Nerve 45: 796-802, 2012.

13. Birnkrant DJ, Bushby K, Bann CM, Apkon SD, Blackwell A, Colvin MK, Cripe L, Herron AR, Kennedy A, Kinnett K, et al: Diagnosis and management of Duchenne muscular dystrophy, part 3: Primary care, emergency management, psychosocial care, and transitions of care across the lifespan. Lancet Neurol 17: 445-455, 2018

14. Tandon A, Villa CR, Hor KN, Jefferies JL, Gao Z, Towbin JA, Wong BL, Mazur W, Fleck RJ, Sticka JJ, et al: Myocardial fibrosis burden predicts left ventricular ejection fraction and is associated with age and steroid treatment duration in Duchenne muscular dystrophy. J Am Heart Assoc 4: e001338, 2015.

15. Sawnani H, Horn PS, Wong B, Darmahkasih A, Rybalsky I, Shellenbarger KC, Tian C, Rutter MM, Simakajornboon N, Amin R, et al: Comparison of pulmonary function decline in steroid-treated and steroid-naïve patients with Duchenne muscular dystrophy. J Pediatr 210: 194-200.e2, 2019.

16. Annexstad EJ, Fagerheim T, Holm I and Rasmussen M: Molecular and clinical characteristics of a national cohort of paediatric Duchenne muscular dystrophy patients in Norway. J Neuromuscul Dis 6: 349-359, 2019.

17. Qi B, Jing HU, Zhe Zhao, Shen Hong Rui and Na LI: Clinical analysis of 155 patients with Duchenne muscular dystrophy. Chin J Contemp Neurol Neurosurg 15: 380-386, 2015.

18. Hu J, Ye Y, Kong M, Hong S, Cheng L, Wang Q, Qin J, Zou L and Jiang L: Daily prednisone treatment in Duchenne muscular dystrophy in southwest China. Muscle Nerve 52: 1001-1007, 2015.

19. Wong BL, Rybalsky I, Shellenbarger KC, Tian C, McMahon MA, Rutter MM, Sawnani H and Jefferies JL: Long-term outcome of interdisciplinary management of patients with Duchenne muscular dystrophy receiving daily glucocorticoid treatment. J Pediatr 182: 296-303.e1, 2017.

20. Sato Y, Yamauchi A, Urano M,Kondo E and Saito K: Corticosteroid therapy for Duchenne muscular dystrophy: Improvement of psychomotor function. Pediatr Neurol 50: 31-37, 2014

21. Ishigaki K, Kato I, Murakami T, Saito T, Sato T, Kajino S, Nakanishi $\mathrm{T}$ and Osawa M: Long-term and low-dose steroid therapy for cardiomyopathy in Duchenne muscular dystrophy patients. J Tokyo Women's Med Coll 83: E14-E19, 2013.

22. Connolly AM, Zaidman CM, Golumbek PT, Cradock MM, Flanigan KM, Kuntz NL, Finkel RS, McDonald CM, Iannaccone ST, Anand P, et al: Twice-weekly glucocorticosteroids in infants and young boys with Duchenne muscular dystrophy. Muscle Nerve 59: 650-657, 2019. 
23. Escolar DM, Hache LP, Clemens PR, Cnaan A, McDonald CM, Viswanathan V, Kornberg AJ, Bertorini TE, Nevo Y, Lotze T, et al: Randomized, blinded trial of weekend vs. daily prednisone in Duchenne muscular dystrophy. Neurology 77: 444-452, 2011.

24. Crabtree NJ, Adams JE, Padidela R, Shaw NJ, Högler W, Roper H, Hughes I, Daniel A and Mughal MZ: Growth, bone health and ambulatory status of boys with DMD treated with daily vs. intermittent oral glucocorticoid regimen. Bone 116: 181-186, 2018.

25. Abutaleb AR, McNally EM, Khan SS, Anderson AS, Carr JC and Wilcox JE: Myocarditis in Duchenne muscular dystrophy after changing steroids. JAMA Cardiol 3: 1006-1010, 2018.

26. Griggs RC, Miller JP, Greenberg CR, Fehlings DL, Pestronk A, Mendell JR, Moxley RT III, King W, Kissel JT, Cwik V, et al: Efficacy and safety of deflazacort vs prednisone and placebo for Duchenne muscular dystrophy. Neurology 87: 2123-2131, 2016.

27. Matthews E, Brassington R, Kuntzer T, Jichi F and Manzur AY: Corticosteroids for the treatment of Duchenne muscular dystrophy. Cochrane Database Syst Rev: CD003725, 2016.

28. McDonald CM, Gordish-Dressman H, Henricson EK, Duong T, Joyce NC, Jhawar S, Leinonen M, Hsu F, Connolly AM, Cnaan A, et al: Longitudinal pulmonary function testing outcome measures in Duchenne muscular dystrophy: Long-term natural history with and without glucocorticoids. Neuromuscul Disord 28 897-909, 2018

29. Pane M, Fanelli L, Mazzone ES, Olivieri G, D'Amico A, Messina S, Scutifero M, Battini R, Petillo R, Frosini S, et al: Benefits of glucocorticoids in non-ambulant boys/men with Duchenne muscular dystrophy: A multicentric longitudinal study using the performance of upper limb test. Neuromuscul Disord 25: 749-753, 2015.

30. Karimzadeh $\mathrm{P}$ and Ghazavi A: Comparison of deflazacort and prednisone in Duchenne muscular dystrophy. Iran J Child Neurol 6: 5-12, 2012.

31. Moxley RT III, Pandya S, Ciafaloni E, Fox DJ and Campbell K: Change in natural history of Duchenne muscular dystrophy with long-term corticosteroid treatment: Implications for management. J Child Neurol 25: 1116-1129, 2010.

32. Matsumura T, Saito T, Fujimura H, Shinno S and Sakoda S: A longitudinal cause-of-death analysis of patients with Duchenne muscular dystrophy. Rinsho Shinkeigaku 51: 743-750, 2011 (In Japanese).

33. Al-Zougbi A, Mathews KD and Shibli-Rahhal A: Use of bone age for evaluating bone density in patients with Duchenne muscular dystrophy: A preliminary report. Muscle Nerve 59: 422-425, 2019

34. Ma J, McMillan HJ, Karagüzel G, Goodin C, Wasson J, Matzinger MA, DesClouds P, Cram D, Page M, Konji VN, et al: The time to and determinants of first fractures in boys with Duchenne muscular dystrophy. Osteoporos Int 28: 597-608, 2017.
35. Lim KR, Maruyama R and Yokota T: Eteplirsen in the treatment of Duchenne muscular dystrophy. Drug Des Devel Ther 11: 533-545, 2017.

36. Conklin LS, Damsker JM, Hoffman EP, Jusko WJ, Mavroudis PD, Schwartz BD, Mengle-Gaw LJ, Smith EC, Mah JK, Guglieri M, et al: Phase IIa trial in Duchenne muscular dystrophy shows vamorolone is a first-in-class dissociative steroidal anti-inflammatory drug. Pharmacol Res 136: 140-150, 2018.

37. Kumaki D, Nakamura Y, Sakai N, Kosho T, Nakamura A, Hirabayashi S, Suzuki T, Kamimura M and Kato H: Efficacy of denosumab for glucocorticoid-induced osteoporosis in an adolescent patient with Duchenne muscular dystrophy: A case report. JBJS Case Connect 8: e22, 2018.

38. Nagy S, Hafner P, Schmidt S, Rubino-Nacht D, Schädelin S, Bieri $\mathrm{O}$ and Fischer D: Tamoxifen in Duchenne muscular dystrophy (TAMDMD): Study protocol for a multicenter, randomized, placebo-controlled, double-blind phase 3 trial. Trials 20: 637, 2019

39. Buyse GM,Voit T,Schara U,StraathofCS,D'Angelo MG,BernertG, Cuisset JM, Finkel RS, Goemans N, McDonald CM, et al: Efficacy of idebenone on respiratory function in patients with Duchenne muscular dystrophy not using glucocorticoids (DELOS): A double-blind randomised placebo-controlled phase 3 trial. Lancet 385: 1748-1757, 2015.

40. Victor RG, Sweeney HL, Finkel R, McDonald CM, Byrne B, Eagle M, Goemans N, Vandenborne K, Dubrovsky AL, Topaloglu H, et al: A phase 3 randomized placebo-controlled trial of tadalafil for Duchenne muscular dystrophy. Neurology 89: 1811-1820, 2017.

41. Wood CL, Cheetham TD, Guglieri M, Bushby K, Owen C, Johnstone $\mathrm{H}$ and Straub V: Testosterone treatment of pubertal delay in Duchenne muscular dystrophy. Neuropediatrics 46: 371-376, 2015.

42. Perera N, Sampaio H, Woodhead H and Farrar M: Fracture in Duchenne muscular dystrophy: Natural history and vitamin D deficiency. J Child Neurol 31: 1181-1187, 2016.

43. Srinivasan R, Rawlings D, Wood CL, Cheetham T, Moreno AC Mayhew A, Eagle M, Guglieri M, Straub V, Owen C, et al: Prophylactic oral bisphosphonate therapy in duchenne muscular dystrophy. Muscle Nerve 54: 79-85, 2016.

This work is licensed under a Creative Commons Attribution-NonCommercial-NoDerivatives 4.0 International (CC BY-NC-ND 4.0) License. 